\title{
Associations between dietary macronutrients and blood lipids in the UK Biobank study
}

\author{
R.K. Kelly ${ }^{1}$, C. Watling ${ }^{1}$, T.Y.N. Tong ${ }^{1}$, C. Piernas ${ }^{2}$, G. Fensom ${ }^{1}$, J. Carter ${ }^{3}$, K. Papier ${ }^{1}$, \\ T.J. Key ${ }^{1}$ and A. Perez-Cornago ${ }^{1}$ \\ ${ }^{1}$ Cancer Epidemiology Unit, Nuffield Department of Population Health, University of Oxford, Richard Doll Building, \\ Oxford OX3 7LF, United Kingdom, \\ ${ }^{2}$ Nuffield Department of Primary Care Health Sciences, University of Oxford, Oxford, United Kingdom and \\ ${ }^{3}$ Clinical Trial Service Unit and Epidemiological Studies Unit, University of Oxford, Oxford, United Kingdom
}

Blood lipids are established risk factors for cardiovascular disease. However, the relationship between dietary macronutrients and blood lipids remains unclear and there is little evidence regarding the association between different dietary sources of macronutrients and blood lipids. Therefore, we aimed to investigate the associations between macronutrients and sources of macronutrients and blood lipids in the UK Biobank study. ${ }^{(1)}$

We studied 27,587 participants with average dietary intake data from $\geq$ two (maximum of five) 24-hour dietary assessments including one assessment at baseline. ${ }^{(2)}$ Non-fasting total cholesterol, low-density lipoprotein cholesterol (LDL-C), high-density lipoprotein cholesterol (HDL-C), triglycerides, apolipoprotein B (ApoB) and apolipoprotein A1 (ApoA1) were measured in serum collected at baseline. Multivariable linear regressions were conducted to calculate geometric mean concentrations $(95 \%$ confidence interval (Cis)) of blood lipids according to quintiles of macronutrient intakes. Tests for linear trend were performed using quintiles of macronutrients as continuous variables in the regression model. After applying Bonferroni correction for multiple comparisons (for 26 exposures, $\mathrm{p}<0.002$ ), most results were statistically significant and therefore, associations were described only if there was $>4 \%$ difference in blood lipids between the lowest and highest quintiles of macronutrient intake.

Intake of free sugars was positively associated with triglycerides $(0.15 \mathrm{mmol} / \mathrm{L}$ difference in geometric mean between highest and lowest quintile of intake; $95 \% \mathrm{CI} ; 0.12$ to $0.17 \mathrm{mmol} / \mathrm{L}$; p-trend $<0.0001)$ and inversely associated with $\mathrm{HDL}-\mathrm{C}(-0.07$; -0.08 to $-0.06 ; \mathrm{p}$-trend $<0.0001)$. Intake of non-free sugars was inversely associated with triglycerides $(-0.07 ;-0.10$ to $-0.05 ; \mathrm{p}$-trend $<$ $0.0001)$. Higher intake of saturated fatty acids (SFA) was positively associated with LDL-C $(0.17 ; 0.14$ to $0.20 ; \mathrm{p}$-trend $<0.0001)$ and higher intake of omega-3 fatty acids was positively associated with HDL-C $(0.06 ; 0.05$ to 0.07 ; p-trend $<0.0001)$. Moreover, intakes of omega-3 fatty acids $(-0.14 ;-0.17$ to -0.12 ; p-trend $<0.0001)$, omega- 6 fatty acids $(-0.06 ;-0.08$ to -0.04 , p-trend $<0.0001)$ and monounsaturated fatty acids $(-0.06 ;-0.09$ to -0.04 , p-trend $<0.0001)$ were inversely associated with triglycerides. Intake of fat from animal sources was positively associated with HDL-C $(0.06 ; 0.05$ to 0.07 , p-trend $<0.0001)$ and inversely associated with triglycerides $(-0.07 ;-0.09$ to -0.04 ; p-trend $<0.0001)$. Intake of protein from animal sources was inversely associated with triglycerides $(-0.07 ;-0.10$ to -0.05 ; p-trend $<0.0001)$. Alcohol intake of $>30$ grams per day compared to $<1$ gram per day was associated with higher HDL-C $(0.22 ; 0.20$ to 0.23 ; p-trend $<0.001)$ and lower triglycerides $(-0.14 ;-0.16$ to -0.12 ; p-trend $<0.0001)$. Similar directions of associations with LDL-C and HDL-C were observed for ApoB and ApoA1, respectively.

Findings that intake of free sugars and non-free sugars relate differently with blood lipids indicate that the quality of dietary carbohydrate consumed can have important implications for CVD prevention. Further, this study confirms existing evidence for associations between SFA and LDL-C. Associations between protein intake from animal sources and alcohol and triglycerides require further investigation.

1. Sudlow, C, Gallacher J, Allen, N et al. (2015) PLos Med 12, 1-10

2. Liu, B, Young, H, Crowe FL et al. (2011) Public Health Nutr 14, 1998-2005 